\title{
Eye Gaze Correlates of Motor Impairment in VR Observation of Motor Actions
}

\author{
J. Alves ${ }^{1}$; A. Vourvopoulos ${ }^{1}$; A. Bernardino ${ }^{2}$; S. Bermúdez i Badia ${ }^{1}$ \\ ${ }^{1}$ Madeira Interactive Technologies Institute, Universidade da Madeira, Funchal, Portugal; \\ ${ }^{2}$ Instituto de Sistemas e Robótica, Instituto Superior Técnico, Lisboa, Portugal
}

\section{Keywords}

Action observation, eye movements, stroke, virtual reality

\section{Summary}

Introduction: This article is part of the Focus Theme of Methods of Information in Medicine on "Methodologies, Models and Algorithms for Patients Rehabilitation".

Objective: Identify eye gaze correlates of motor impairment in a virtual reality motor observation task in a study with healthy participants and stroke patients.

Methods: Participants consisted of a group of healthy subjects $(\mathrm{N}=20)$ and a group of stroke survivors $(N=10)$. Both groups were required to observe a simple reach-and-grab and place-and-release task in a virtual environment. Additionally, healthy subjects were required to observe the task in a normal condition and a constrained movement condition. Eye movements were recorded during the observation task for later analysis.
Results: For healthy participants, results showed differences in gaze metrics when comparing the normal and arm-constrained conditions. Differences in gaze metrics were also found when comparing dominant and non-dominant arm for saccades and smooth pursuit events. For stroke patients, results showed longer smooth pursuit segments in action observation when observing the paretic arm, thus providing evidence that the affected circuitry may be activated for eye gaze control during observation of the simulated motor action.

Conclusions: This study suggests that neural motor circuits are involved, at multiple levels, in observation of motor actions displayed in a virtual reality environment. Thus, eye tracking combined with action observation tasks in a virtual reality display can be used to monitor motor deficits derived from stroke, and consequently can also be used for rehabilitation of stroke patients.

Correspondence to:

Júlio Alves

Madeira-ITI

Polo Científico e Tecnológico da Madeira, Floor -2

Caminho da Penteada

9020-105 Funchal

Portugal

E-mail: juliomalves@gmail.com
Methods Inf Med 2016; 55: 79-83

http://dx.doi.org/10.3414/ME14-01-0125

received: November 26, 2014

accepted: October 6, 2015

epub ahead of print: December 7, 2015

\section{Introduction}

Stroke is one of the primary causes of permanent disability among the current population [1]. For this reason, the mechanisms underlying stroke recovery have been the focus of several studies that showed the importance of brain plasticity in the recovery process $[2,3]$. Some researchers proposed the recruitment of mirror neurons for stroke rehabilitation [4]. This paper proposes the use of eye tracking technology to quantify the recovery process, by exploiting the neural correlates of eye gaze trajectories in the observation and execution of goal directed actions in a virtual reality display.

Mirror neurons are single neurons observed in primates that fire both when ob- serving behaviors performed by others (action observation) or when the individual plans his/her own actions (action execution) $[5,6]$. Research has shown that mirror neurons may serve recognition and imitation of goal-directed actions, and that these neurons are crucial to social interactions, providing the basis for understanding the actions of others through the connection between action and perception [6-9]. Through neuroimaging techniques, researchers have been able to locate specific areas of human brain activation and determine the spatial and temporal congruency between observing and executing actions [10, 11]. However, no existing model allows us to fully understand the shared neural mechanisms between observation and execution, and propose how to maximally exploit it in motor rehabilitation training.

There are multiple models of the mirror neurons and their function, but there is a lack of an integrative understanding of their function [12]. However, most place the mirror neurons at the intersection of action observation and action execution. According to Oztop et al. [9] the dual activation of mirror neurons during observation/execution is explained by two processes: i) automatic engagement of mental state inference during action observation, and ii) forward prediction by the mirror neurons for motor control during action execution. Because eye gaze is tightly coupled to sensory prediction during both action observation and action execution [13], the measurement of eye movements has become a promising method for quantifying multiple aspects of cognition [14]. Studies have demonstrated congruency in gaze metrics between action execution and action observation, supporting the idea 
that these processes have a partially shared neural network [15]. From a rehabilitation standpoint, some studies have demonstrated strong evidence that action observation has a positive effect on rehabilitation of motor deficits after stroke [16-18]. Others $[19,20]$ have shown the feasibility of using eye tracking in neurorehabilitation. With the increasing availability of low-cost devices, eye gaze will play an important role in rehabilitation and diagnostics.

In this study we take advantage of the assumed shared neural mechanisms in action observation and execution to explore their potential in rehabilitation. We propose a novel technology that assesses eye gaze behavior in a virtual reality (VR) observation task. We demonstrate its use in healthy subjects as well as in stroke patients, suggesting important implications for diagnostic and rehabilitation purposes.

\section{Objectives}

The objective of this study is to assess eye gaze behavior in a VR observation task in healthy participants, who represent an intact mirror neuron system, and stroke patients, whose mirror neurons may potentially be damaged. The eye gaze of participants is analyzed in a task where subjects observe an arm in a virtual environment while executing reaching and grasping actions. We aim at verifying the following hypotheses:

a) existence of differences in gaze metrics in healthy participants using their dominant arm when compared to their non-dominant arm during action observation, due to interference of arm dominance during the task;

b) existence of differences in gaze metrics in healthy participants during normal condition versus impaired sensorimotor condition while observing the task. Since action and perception are closely coupled through sensorimotor maps, impairing motor action in healthy participants may also affect their motor planning, and subsequently their gaze patterns, during the task;

c) Existence of differences in gaze metrics in stroke patients using their paretic arm when compared to their nonparetic arm during action observation, due to the recruitment of the motor control areas affected by stroke.

Experiments were conducted with healthy participants and stroke patients, using a virtual environment as stimulus and eye tracking technology for data acquisition.

\section{Methods}

\subsection{Participants}

For the healthy group, 20 participants ( $3 \mathrm{fe}$ male and 17 male) were recruited with a mean age of 30.4 years ( $\mathrm{SD}=6.5$ years). All but one participant were right handed. For the stroke patients group, 10 stroke survivors (5 male, 5 female), with a mean age of 66.1 years $(S D=10.6$ years) and a mean of 221.2 days after stroke (SD = 157.4 days), participated in the study. Seven of these patients suffered an ischemic stroke and three patients suffered an intra-cerebral hemorrhage. Four patients had a left-sided brain lesion and six patients had a right-sided lesion. Patients with no arm mobility and/or with severe attention deficits were excluded from the study. Stroke patients were recruited from Hospital Dr. Nélio Mendonça and Hospital Dr. João de Almada, located in the city of Funchal, Portugal. Participants in both groups were naive to the system and hypotheses being tested. All of them supplied written informed consent prior to participation. The study was approved by the Ethical Committee of the Regional Health System of Madeira (SESARAM).

\subsection{System}

A custom VR task was developed using the Unity 3D game engine (Unity Technologies, San Francisco, USA). The VR environment was displayed on a 4:3 monitor $(1024 \times 768$ pixels resolution) with an integrated eye tracking system, the Tobii T120 Eye Tracker (Tobii Technology, Stockholm, Sweden). Eye movements were recorded at a sampling rate of $60 \mathrm{~Hz}$. A laptop computer connected to the eye tracker ran the custom VR software during the trials. Eye tracking data were acquired, logged and sent to VR through the RehabNet Control Panel. RehabNetCP integrates a large number of commercial and experimental interface devices to enable the patient-task interaction within VR, developed for motor and cognitive neurorehabilitation in both a clinical and non-clinical environment [21].

Participants were placed in front of the eye tracking system, with their head at about $60 \mathrm{~cm}$ distance from the screen, and with both hands over the table in front of them ( $\triangleright$ Figure 1$)$. The VR environment, shown in the eye tracker display, presented the user with a virtual arm that performed a sequence of movements.

\subsection{Procedure}

Participants were presented with a simple reach-and-grab and place-and-release task in the virtual environment ( $\triangleright$ Figure 1). The environment was presented in a firstperson perspective, allowing the virtual arm to be consistent with the participant's point of view. The task consisted of grabbing a virtual ball (either with a left or right virtual arm), moving it to a target destination (which would make the ball disappear), then come back to the initial position and wait 3 seconds for the task to restart. There were four predefined points for the ball's initial position, all equidistant to the target and horizontally symmetric.

Both groups were presented with two different conditions, in the following order: i) action observation - the participants were required to observe, for posterior repetition, a pre-recorded execution of the virtual arm grabbing the ball and taking it to the target destination; and ii) action execution with eye gaze - the participants were required to actively grab the ball with the virtual arm using their eye gaze and to take it to the target destination. In addition, healthy participants had to perform these two conditions twice, in a normal situation and a constrained-induced movement situation. For each condition, each participant had to perform (or observe) 40 repetitions of the task for each arm, with each repetition lasting around $5 \mathrm{~s}$. The order of the initial position of the virtual ball was chosen randomly (out of the four predefined positions) for every repetition, making sure 

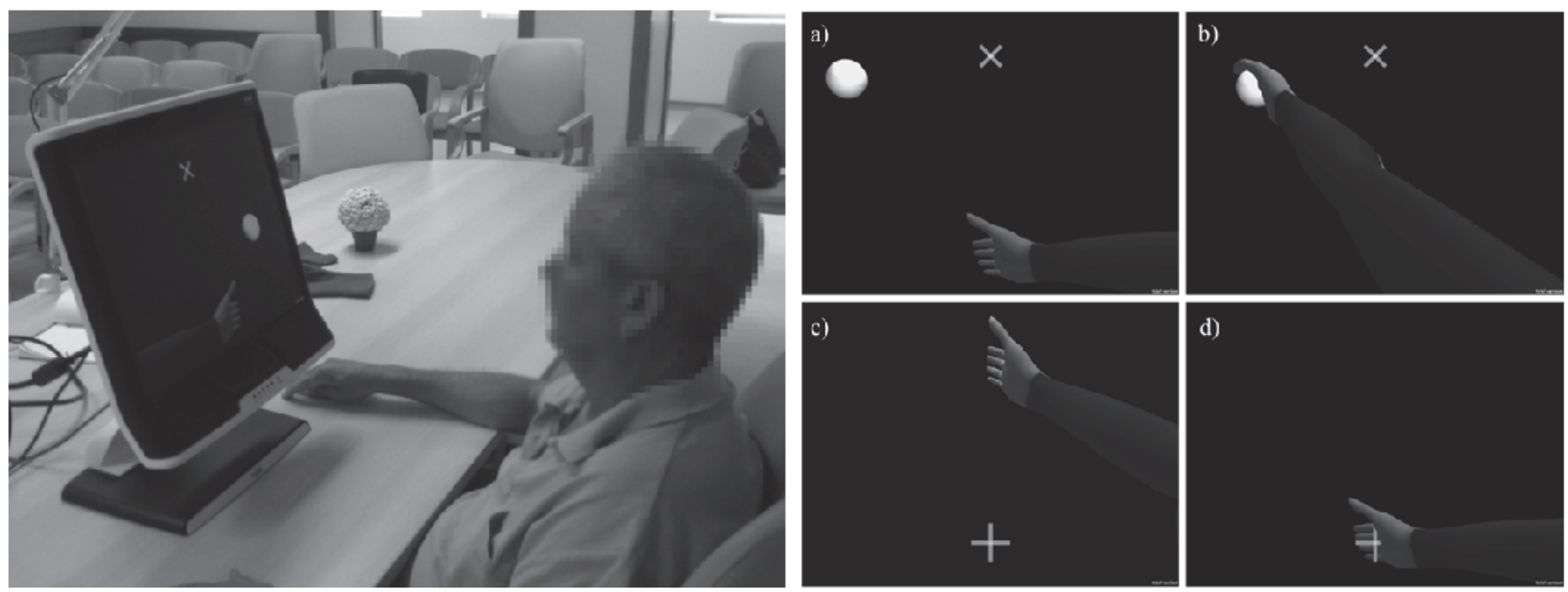

Figure 1 Experimental setup being used by a stroke patient, consisting of a monitor with an integrated eye tracker running a custom made VR task. The task consist of four steps: a) reaching and grasping of a virtual ball, b) placing it at the target, c) releasing it, and d) moving back to initial position

that all initial positions were presented 10 times. In this paper we focus on the analysis of condition i). The results of ii), already reported in our previous work [18], did not show strong correlations with arm motor deficits and thus are not included in this paper.

\subsection{Data Analysis}

All data analysis was performed with Matlab (MathWorks Inc., Natick, MA, USA). Eye tracking data was temporally smoothed with a Gaussian window of 1.6 seconds with $\mathrm{SD}=0.16 \mathrm{~s}$, and converted to screen coordinates $(\mathrm{X}, \mathrm{Y})$. Resting periods and segments with missing data were removed from the analysis. According to the velocity profile of the data, eye tracking behavior was classified into 1) fixations, 2) saccadic movements, and 3) smooth pursuit. For each behavior detected, the number of occurrences and their duration were assessed. In addition, the accumulated travelled distance was also computed.

Out of the 10 stroke participants, one dataset of the action observation condition was corrupt. The 2-sided Lilliefor test revealed that data was not normally distributed. To test against different conditions where size group data differ in size the non-parametric Mann-Whitney test was used. A non-parametric matched pairs Wilkoxon test was used to assess differ- ences between paretic and non-paretic data for the stroke patient data - and to assess differences between dominant and nondominant data, and between constrained and non-constrained conditions for the healthy participant data.

\section{Results}

\subsection{Gaze Density Maps}

The distribution of gaze patterns (fixations, saccadic movement, smooth pursuit) in action observation was assessed in the healthy group, for the normal and constrained conditions, and in the stroke patients group ( $\triangleright$ Figure 2).

There is consistency when comparing eye gaze patterns between the two experimental conditions for healthy participants and stroke patients. Fixations are mostly clustered around the targets (release place at the top-center and resting position at the bottom-center of the screen) or virtual objects (two on the right and two on the left halves of the screen) as shown in $>$ Figure 1. Saccadic movements were detected mostly between the target position and the resting position. Because these two elements are at opposite ends of the screen they generate more saccadic movements. Smooth movements are detected mostly in the areas between virtual objects and their respective targets. We did not observe major differences in the distribution of eye gaze patterns when comparing the different conditions.

\subsection{Gaze Metrics}

For the next analysis, the following gaze metrics were extracted from the eye tracking data: number of fixations, number of saccades, number of smooth pursuit segments, duration of fixations, duration of saccades, and duration of smooth pursuit segments ( $\triangleright$ Table 1$)$.

When performing a within subject analysis to the different eye gaze patterns in healthy participants in the normal observation conditions, results revealed shorter saccades when observing the dominant arm $(M d n=265 \mathrm{~ms})$ than when observing the non-dominant $\operatorname{arm}(M d n=291 \mathrm{~ms})$, $\mathrm{T}=31, \mathrm{p}<0.01$, and less smooth pursuit events when observing the dominant arm $(M d n=314)$ compared to the non-dominant arm $(M d n=379), \mathrm{T}=37, \mathrm{p}<0.05$. No significant differences were found between dominant and non-dominant arm in the movement constrained condition. In the case of stroke patients, the within subject analysis revealed longer smooth pursuit when observing the paretic arm $(M d n=587$ ms) than when observing the non-paretic $\operatorname{arm}(M d n=567 \mathrm{~ms}), \quad \mathrm{T}=154$, $\mathrm{p}<0.01$. In average, smooth pursuit in the observation condition was $30 \mathrm{~ms}$ longer. However, no more differences were found in any other eye gaze metric for stroke patients. 


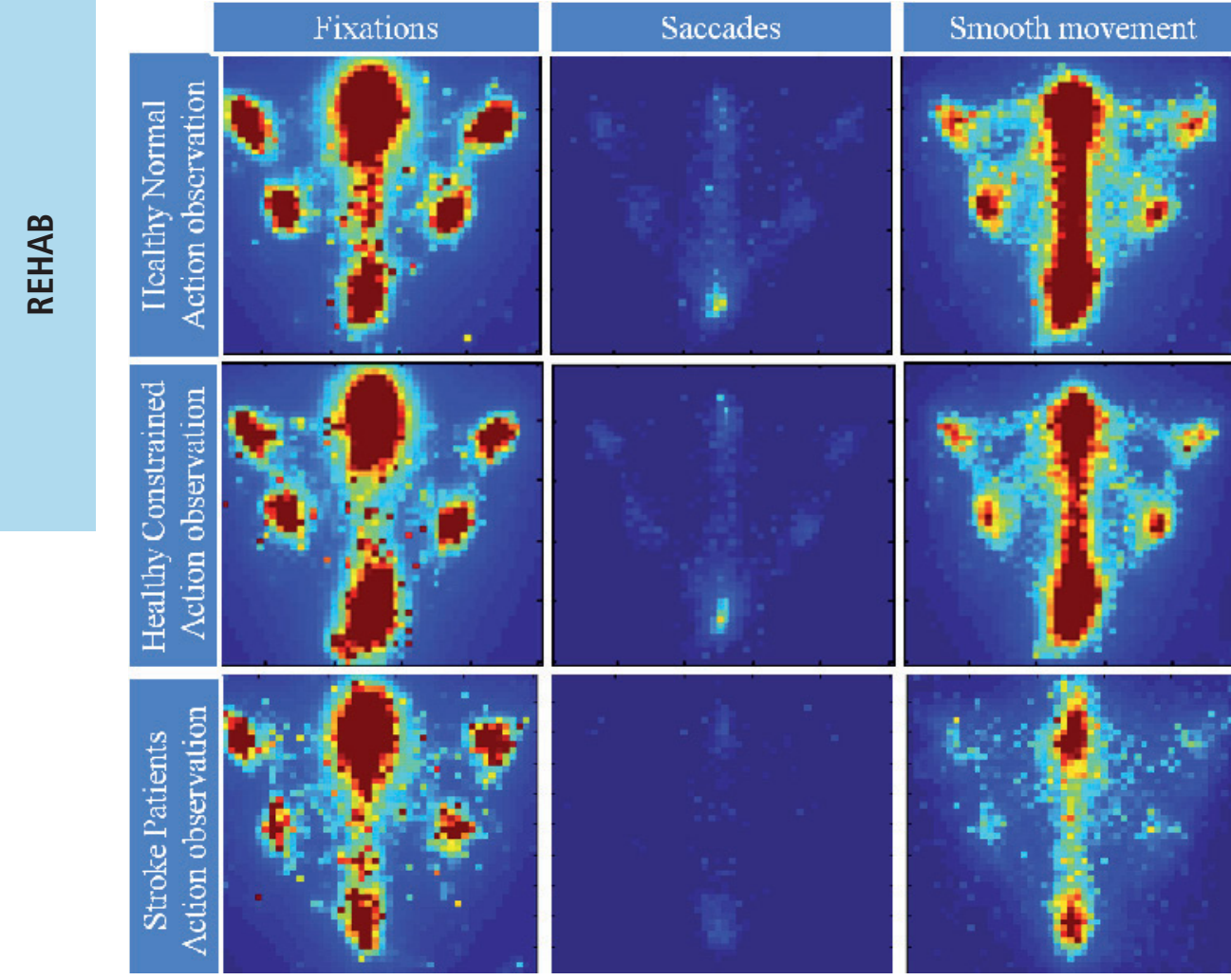

Figure 2 Density maps for action observation according to the detected eye movements in the healthy group and stroke patients group

When comparing the normal condition and movement constrained condition in the healthy group and with the stroke group, it was found that fixations are less likely to occur in the constrained condition $(M d n=1160)$ when compared to the normal condition $(M d n=1286), \mathrm{T}=224$, $\mathrm{p}<0.05$, and with stroke patients $(M d n=$ $1284), \mathrm{U}=682, \mathrm{p}<0.01$. Additionally, differences in the dominant arm were found between normal $(M d n=1309)$ and con- strained conditions $(M d n=1061), \mathrm{T}=34$, $\mathrm{p}<0.05$. The duration of fixations was found to be significantly longer in stroke patients $(M d n=378 \mathrm{~ms})$ than in the normal condition for healthy participants $(M d n=228 \mathrm{~ms}), \mathrm{U}=696, \mathrm{p}<0.01$. Differences were also found for the non-dominant arm between normal $(M d n=210 \mathrm{~ms})$ and constrained conditions $(M d n=$ $174 \mathrm{~ms}), \mathrm{T}=28, \mathrm{p}<0.01$. Finally, less saccades were detected for stroke patients
$(M d n=53)$ than for healthy participants in the normal condition $(M d n=60), \mathrm{U}=370$, $\mathrm{p}<0.05$. No other significant differences were found between conditions and groups.

\section{Discussion and Conclusions}

There is a growing body of research that supports the use of action observation as a valid paradigm for post-stroke rehabilitation due to shared neural mechanisms between execution and observation circuits. In this study we quantified action observation metrics, by means of the combination of VR and eye tracking technology, showing its correlation to execution deficits.

Differences in gaze metrics were found when comparing normal condition with constrained movement condition in fixation count and duration, and with stroke patients in fixation duration and saccades count. Movement constrained condition data and stroke patients were consistent in fixation duration and saccades count. Saccades duration, smooth pursuit count and duration were not modulated by the conditions. However, a handedness effect was detected in the normal condition for saccades duration and smooth pursuit count, and differences between paretic and nonparetic arms were detected in stroke patients for smooth pursuit duration. Hence, data suggests that gaze metrics are differently sensitive to motor impairment, stroke and handedness. This fact supports the results found in stroke patients, by showing

Table 1 Median values of the eye gaze metrics in action observation for each arm, in the healthy group and stroke patients group. ${ }^{(*)}$ indicates significant within condition arm differences. ${ }^{(\mathrm{NC})}$ indicates significant differences between normal and constrained conditions. (NS) indicates significant differences between normal and stroke groups. ${ }^{(C S)}$ indicates significant differences between constrained and stroke groups.

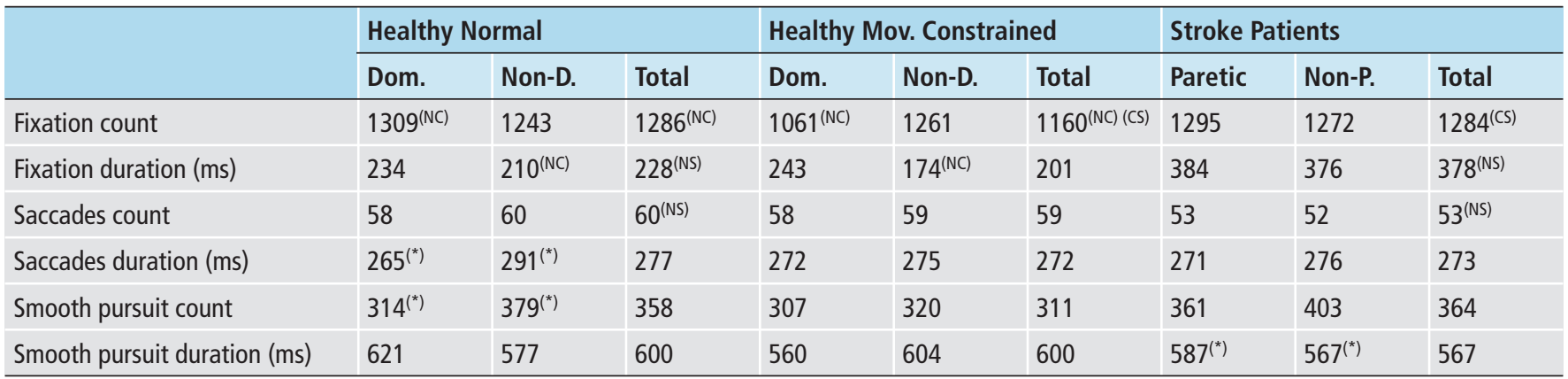


that differences between the paretic and non-paretic arms in the observation condition could not be due to arm dominance, but to some other factor such as the recruitment of motor control areas of the brain affected by stroke.

Consequently, considering the first hypothesis (a), we found differences between dominant versus non-dominant arm only during action observation. Consistent with the second hypothesis $(\mathrm{b})$, the differences shown between the constrained and nonconstrained condition demonstrate that impairing the sensorimotor contingencies of the arm in healthy participants also affects their eye gaze during observation of a goal-oriented task, with some data consistent with stroke data. Considering hypothesis (c), differences were found between paretic and non-paretic arm during action observation, which may be explained by the recruitment of motor control areas of the brain affected by stroke.

The findings of this study strongly suggest that eye tracking combined with a VR action observation task can be used to assess motor deficits derived from stroke, and therefore has a large potential to be used in motor rehabilitation. However, the main limitation of this approach is the widespread neural basis of the motor action-observation loop, and the currently limited understanding of the specific role of the mirror neuron system in it. Thus, lesions of the mirror neuron system may affect multiple aspects of motor execution and observation. At the same time, not all impairments to the motor execution and observation loop are due to lesions to the mirror neuron system. Consequently, more integrative models of the action execution and observation loop considering the mirror neuron system need to be in place in order to understand how lesion location and size disrupt both observation as well as the action execution. Hence, detection of impairment through eye tracking is possible and may be sufficient for the diagnosis and monitoring of some motor deficits, but not a necessary condition since not all impairments of the action execution system may be measurable through eye gaze. This indicates that with our current knowledge, the proposed approach may be more ad- equate for monitoring the restoration of motor action execution and observation systems to normal function. With the increasing appearance of low cost eye tracking devices, treatments aiming at exploiting the shared mechanisms between eye gaze control and action observation can become a cost effective continuous assessment and rehabilitation tool for at home use after hospital discharge. Further, the integration of such an approach to current rehabilitation technologies could provide new insights on how different lesions affect differently eye gaze, allowing researchers to refine and improve theoretical action execution models through the fitting of currently unavailable eye gaze data.

\section{Acknowledgments}

This work is supported by the European Commission through the RehabNet project - Neuroscience Based Interactive Systems for Motor Rehabilitation - EC (303891 RehabNet FP7-PEOPLE2011-CIG), and by the Fundação para Ciência e Tecnologia (Portuguese Foundation for Science and Technology) through the project AHA-Augmented Human Assistance, CMUP-ERI/HCI/ 0046/2013, doctoral grant SFRH/BD/ 97117/2013, and Projeto Estratégico LA 9-2013-2014.

\section{References}

1. Mukherjee D, Patil CG. Epidemiology and the global burden of stroke. World Neurosurgery 2011; 76 (6): S85-S90.

2. Hermann DM, Chopp M. Promoting brain remodelling and plasticity for stroke recovery: therapeutic promise and potential pitfalls of clinical translation. The Lancet Neurology 2012; 11 (4): 369-380.

3. Seitz RJ, Donnan GA. Role of neuroimaging in promoting long-term recovery from ischemic stroke. Journal of Magnetic Resonance Imaging 2010; 32 (4): $756-772$.

4. Rizzolatti G, Sinigaglia C. The functional role of the parieto-frontal mirror circuit: interpretations and misinterpretations. Nature Reviews Neuroscience 2010; 11 (4): $264-274$.

5. Rizzolatti G, Craighero L. The mirror-neuron system. Annu Rev Neurosci 2004; 27: 169-192.

6. Buccino G, Binkofski F, Riggio L. The mirror neuron system and action recognition. Brain and language 2004; 89 (2): 370 -376.
7. Oztop E, Arbib MA. Schema design and implementation of the grasp-related mirror neuron system. Biological cybernetics 2002; 87 (2): 116-140.

8. Bonaiuto J, Rosta E, Arbib MA. Extending the mirror neuron system model. I. Biological cybernetics 2007; 96 (1): 9-38.

9. Oztop E, Kawato M, Arbib MA. Mirror neurons: functions, mechanisms and models. Neuroscience letters 2013; 540: 43-55.

10. Grezes J, Decety J. Functional anatomy of execution, mental simulation, observation, and verb generation of actions: a meta-analysis. Human Brain Mapping 2001; 12 (1): 1-19.

11. Holmes PS, Cumming J, Edwards MG. Movement imagery, observation, and skill. In: Guillot A, Collet $\mathrm{C}$, editors. The neurophysiological foundations of mental and motor imagery. Oxford University Press; 2010. pp 245-269.

12. Thill S, Caligiore D, Borghi AM, Ziemke T, Baldassarre G. Theories and computational models of affordance and mirror systems: an integrative review. Neuroscience \& Biobehavioral Reviews 2013; 37 (3): 491- 521.

13. Brouwer AM, Franz VH, Gegenfurtner KR. Differences in fixations between grasping and viewing objects. Journal of Vision 2009; 9 (1): 18.

14. Liversedge SP, Findlay JM. Saccadic eye movements and cognition. Trends in Cognitive Sciences 2000; 4 (1): 6-14.

15. Causer J, McCormick SA, Holmes PS. Congruency of gaze metrics in action, imagery and action observation. Frontiers in human neuroscience 2013; 7: 604 .

16. Ertelt D, Small S, Solodkin A, Dettmers C, McNamara A, Binkofski F, et al. Action observation has a positive impact on rehabilitation of motor deficits after stroke. Neuroimage 2007; 36: T164-T173.

17. Mulder T. Motor imagery and action observation: cognitive tools for rehabilitation. Journal of neural transmission 2007; 114 (10): $1265-1278$.

18. Alves J, Vourvopoulos A, Bernardino A, Bermudez i Badia S. Eye gaze patterns after stroke: correlates of a VR action execution and observation task. REHAB 2014. Proceedings of the 2nd Patient Rehabilitation Research Techniques Workshop; 2014 May 20; Oldenburg, Germany. ICST; 2014.

19. Amantis R, Corradi F, Molteni AM, Massara B, Orlandi M, Federici S, et al. Eye-tracking assistive technology: Is this effective for the developmental age? Evaluation of eye-tracking systems for children and adolescents with cerebral palsy. In: Gelderblom GJ, Soede M, Adriaens L, Miesenberger K, editors. Everyday Technology for Independence and Care: AAATE 2011. Amsterdam: IOS Press; 2011. pp 489- 496.

20. Loconsole C, Bartalucci R, Frisoli A, Bergamasco $\mathrm{M}$. A new gaze-tracking guidance mode for upper limb robot-aided neurorehabilitation. WHC 2011. Proceedings of the World Haptics Conference, 2011 IEEE; 2011 June 21-24; Istanbul, Turkey. IEEE; 2011. pp 185-190.

21. Vourvopoulos A, Faria AL, Cameirao MS, Bermudez i Badia S. RehabNet: A distributed architecture for motor and cognitive neuro-rehabilitation. HealthCom 2013. Proceedings of the 2013 IEEE 15th International Conference on e-Health Networking, Applications and Services; 2013 October 9-12; Lisbon, Portugal. IEEE; 2013. pp 454- 459. 